\title{
Estudio de la intercara de uniones entre metales disímiles por medio de ultrasonido ${ }^{(\bullet)}$
}

\author{
G.A. López ${ }^{(*)}$, A. López ${ }^{(\dagger)}$, A. Huerta ${ }^{(*)}$ y D. Jaramillo ${ }^{(* *)}$ \\ Resumen Como resultado de las dificultades encontradas para determinar las características de la intercara \\ entre uniones de materiales disímiles, sobre todo aquellas empleadas en los recubrimientos, surge la \\ posibilidad de emplear las técnicas de ultrasonido. Las técnicas basadas en la diferencia de impedan- \\ cia acústica se han usado de manera exitosa para caracterizar las intercaras plástico- aluminio (adhe- \\ ridas) y metal babbit-acero (en cojinetes). En este estudio se analizan, de manera preliminar, las \\ intercaras de pares aluminio-acero y aluminio-plomo obtenidos por medio de soldadura por explosi- \\ vos. Se utilizó un sistema de adquisición de señales digitales ultrasónicas y un transductor de 5 \\ MHz. Los resultados obtenidos hasta el momento indican que la representación de la señal en forma \\ de "VID" proporciona un criterio de aceptación basado en la relación entre las amplitudes de la inter- \\ cara y la superficie posterior, mientras que la señal de "RF" proporciona un criterio de aceptación \\ basado en la forma de la onda. Se concluye que es posible emplear las señales VID y RF para esta- \\ blecer criterios de aceptación de la unión de las intercaras.
}

Palabras clave: Impedancia acústica. Metales disímiles. Amplitudes. Intercaras. Onda.

\section{Study of bonding interfaces within dissimilar metals by means of ultrasonic waves}

\begin{abstract}
As a result of the associated problems to evaluate bonding characteristics of interfaces between dissimilar metals, mainly those cladded, it has been propposed the use of ultrasonic methods. Acoustic impedance methods had been tested succesfully in the study of plastic-aluminium (glued) and babbit-steel (bearing) interfaces. Preliminary results on explosively bonded aluminium-steel and aluminium-lead interfaces are presented. A digital ultrasonic device with a $5 \mathrm{Mhz}$ transducer was used in this study. So far, results have shown that "VID" signal gives an acceptance criterion based on the ratio of amplitudes coming from interface and bottom surface, meanwhile "RF" signal gives the same criterion based on wave form. It is concluded that VID and RF signals could be used to achieve characteristics of the bonded interfaces.
\end{abstract}

Keywords: Acoustic impedance. Dissimilar metals. Amplitudes. Interfaces. Wave form.

\section{INTRODUCCIÓN}

Este estudio plantea la posibilidad de determinar mediante un ensayo no destructivo la calidad de la unión entre metales disímiles. Actualmente, para determinar si una unión entre materiales disímiles

$(\bullet \quad$ Trabajo recibido el día 28 de julio de 1998 y aceptado en su forma final el 15 de marzo de 1999.

(*) Instituto de Investigaciones Metalúrgicas. Edificio "U", Ciudad Universitaria, Francisco J. Mújica C.P. 58060

(**) Instituto Politécnico Nacional. E.S.I.Q.I.E. Departamento de Ingeniería Metalúrgica Apdo. Postal 75-874 83000 México D.F.

$(\dagger)$ In memoriam cumple con los requerimientos del trabajo a que va a ser sometida, se realizan ensayos destructivos como el ensayo de tracción y el ensayo al cizallamiento, para de esta manera dictaminar si cumple los criterios de aceptación. Debido al costo y tiempo que implica realizar estos ensayos destructivos, se propone una técnica ultrasónica que permita ofrecer criterios de aceptación entre las uniones de metales disímiles. Este método tiene la ventaja de constituir un ensayo rápido y que puede ser llevado a cabo en el lugar de trabajo sin requerir de ninguna preparación previa del material (1). Para llevar a cabo este estudio se analizó, por medio de la técnica de ultrasonido, la intercara de dos uniones soldadas 
obtenidas por explosivos, el cual es un proceso de unión entre metales y/o aleaciones en el estado sólido (2). Los pares estudiados fueron de aluminioplomo y aluminio-acero. La morfología de la intercara fue plana, obtenida con una velocidad de detonación de 1.400 y $1.800 \mathrm{~m} / \mathrm{s}$ respectivamente, con el arreglo mostrado en la figura 1. La intercara resultante para cada una de estas uniones se presenta en la figura 2 ( $a$ y b), donde se observa una intercara plana. El objetivo de este estudio es demostrar la viabilidad de la técnica ultrasónica, basado en el cambio de impedancia acústica que ocurre entre los materiales disímiles, aportando criterios para determinar si una unión es satisfactoria o no.

\section{DESARROLLO EXPERIMENTAL}

\subsection{Materiales}

Los materiales utilizados en el presente estudio son: placa de aluminio de $9,6 \mathrm{~mm}$ y una placa de plomo con 2,31 mm de espesor. Se empleó un explosivo a base de nitrato de amonio con una velocidad de detonación de $1.400 \mathrm{~m} / \mathrm{s}$. Las propiedades acústicas para estos materiales se muestran en la tabla I. Asimismo, se utilizó una placa de aluminio con 6,5 $\mathrm{mm}$ de espesor unida por explosivos a una placa de acero bajo carbono de $4 \mathrm{~mm}$. Se empleó el mismo tipo de explosivo con una velocidad de detonación de $1.800 \mathrm{~m} / \mathrm{s}$ para obtener la intercara plana. Las propiedades acústicas de estos materiales se muestran en la tabla II.
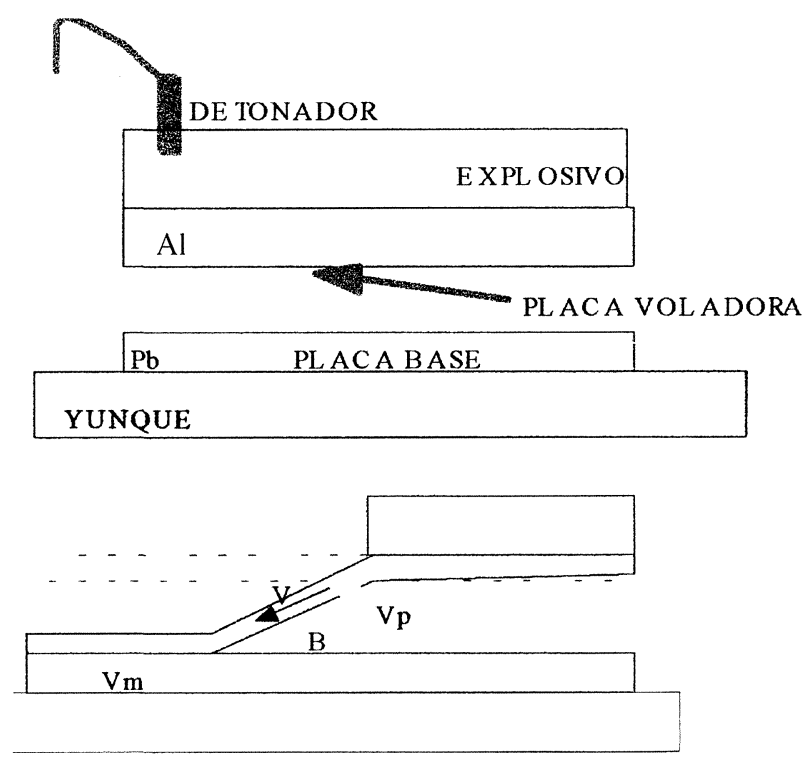

FIG. 1.- Arreglo en paralelo para realizar soldaduras por explosivos

FIG. 1.- Adjustment in parallel for welding by explosive

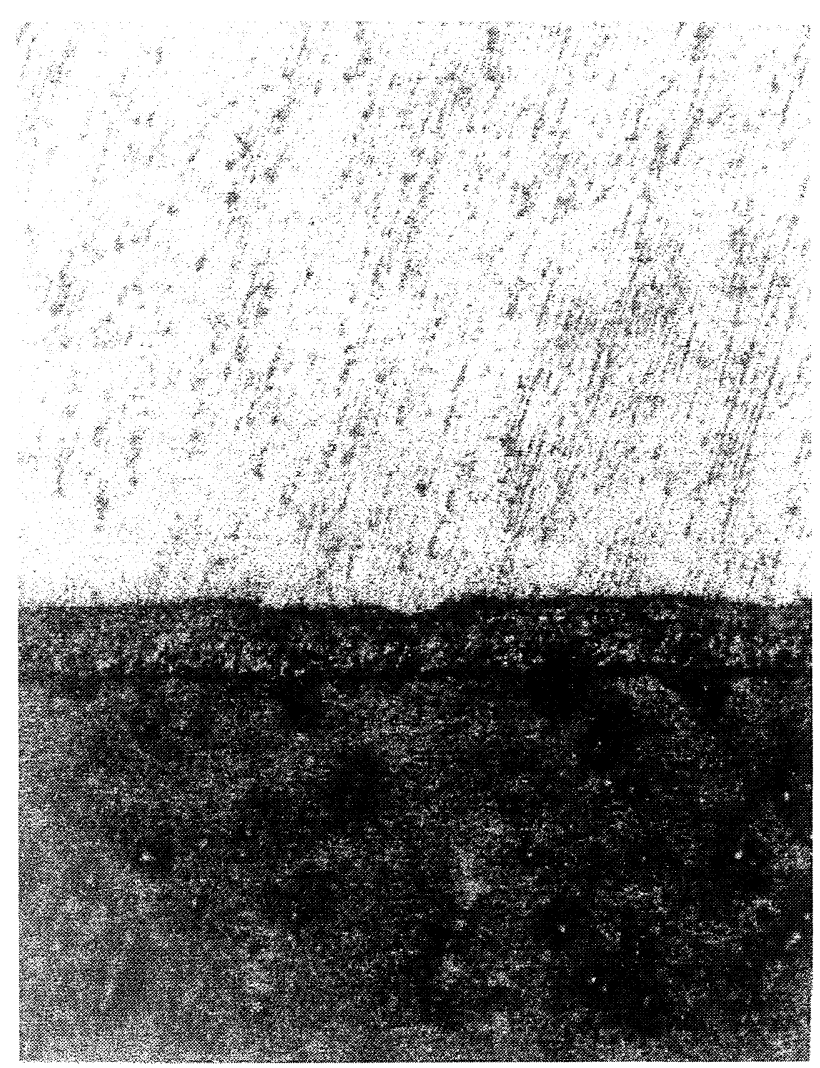

FIG. 2.- a) Intercara plana de soldadura por explosivos de placas de $\mathrm{Al}-\mathrm{Pb}$

FIG. 2.- a) Plane interface from explosively welded plates of $\mathrm{Al}-\mathrm{Pb}$

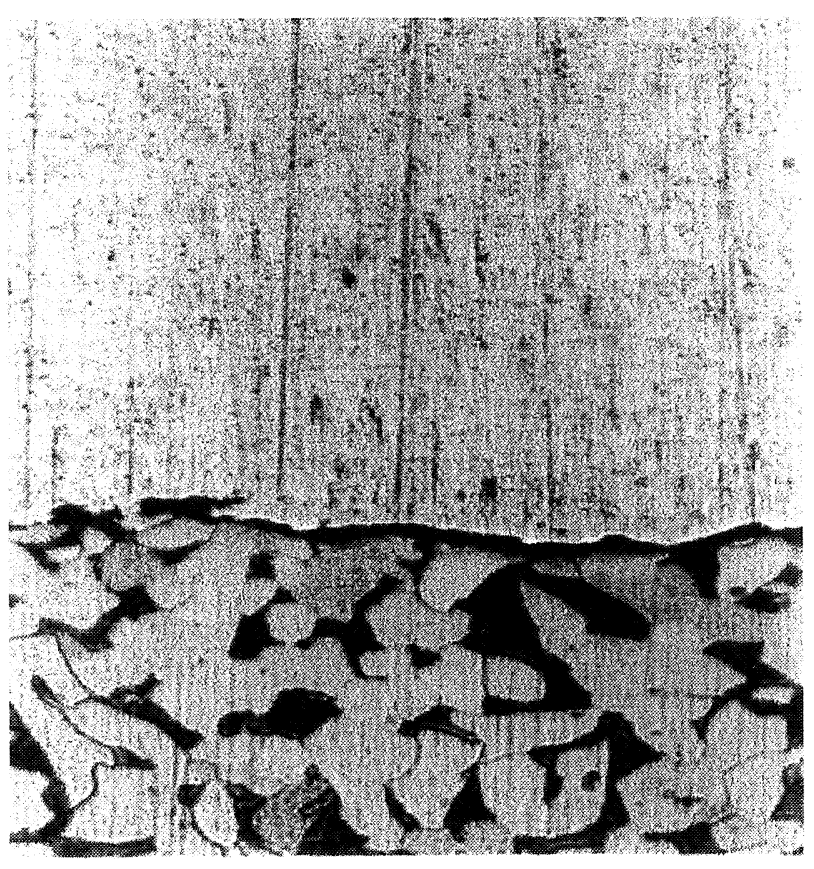

FIG. 2.- b) Intercara plana de soldadura por explosivos de placas de $\mathrm{Al}-\mathrm{Fe}$

FIG. 2.-b) Plane interface from explosively welded plates of $\mathrm{Al}-\mathrm{Fe}$ 
TABLA I.- Propiedades acústicas de Al-Pb

TABLE I.-Acoustic properties of Al-Pb

\begin{tabular}{|l|l|l|l|l|}
\hline METAL & $\mathrm{V}_{\mathrm{L}}(\mathrm{m} / \mathrm{s})$ & $\mathrm{Z}\left(\mathrm{kg} / \mathrm{m}^{2} \mathrm{~s}\right)$ & $\mathrm{t}(\mathrm{ms})$ & $\begin{array}{c}\text { Coeficientes } \\
\%\end{array}$ \\
\hline $\mathrm{Al}$ & 6.320 & 17,06 & 3.037 & $\mathrm{R}=17,8$ \\
$\mathrm{~Pb}$ & 2.160 & 24,49 & 2.390 & $\mathrm{~T}=82,2$ \\
\hline
\end{tabular}

$\mathrm{V}_{\mathrm{L}}(\mathrm{m} / \mathrm{s})=$ Velocidad acústica longitudinal (Acoustic velocity) $\mathrm{Z}\left(\mathrm{kg} / \mathrm{m}^{2} \mathrm{~s}\right)=$ Impedancia acústica (Acoustic impedance) $\mathrm{t}(\mathrm{ms})=$ Tiempo de recorrido (Time of flight)

$\mathrm{R}=$ Coeficiente de Reflexión (Reflection coefficient)

$\mathrm{T}=$ Coeficiente de Transmisión (Transmission coefficient)

TABLA II.- Propiedades acústicas de Al-Fe

TABLE II.-Acoustic properties of Al-Fe

\begin{tabular}{|l|l|l|l|l|}
\hline METAL & $\mathrm{V}_{\mathrm{L}}(\mathrm{m} / \mathrm{s})$ & $\mathrm{Z}\left(\mathrm{kg} / \mathrm{m}^{2} \mathrm{~s}\right)$ & $\mathrm{t}(\mathrm{ms})$ & $\begin{array}{l}\text { Coeficientes } \\
\%\end{array}$ \\
\hline $\mathrm{Al}$ & 6.320 & 17,06 & 2.065 & $\mathrm{R}=45,5$ \\
$\mathrm{Fe}$ & 5.830 & 45,63 & 5.104 & $\mathrm{~T}=54,5$ \\
\hline
\end{tabular}

\subsection{Técnica ultrasónica}

La técnica empleada se basa en el viaje del haz ultrasónico. Cuando este golpea el límite de un segundo material, parte de la energía se refleja (representándose como un eco en la pantalla del osciloscopio) y parte se transmite al segundo material. La característica que determina la cantidad de energía transmitida y reflejada es la impedancia acústica de los materiales unidos (3). Por lo tanto, la impedancia acústica será el factor decisivo para determinar la unión entre materiales disímiles.

La técnica ultrasónica que se utilizó fue de contacto, el método fue de impulso-eco, el cual consiste en que pulsos cortos de vibraciones ultrasónicas son transmitidos en el material de prueba en intervalos pequeños de tiempo. Si el pulso encuentra en su camino una superficie reflectora (con una impedancia acústica diferente) se refleja entonces alguna parte de la energía. La cantidad de energía reflejada depende de la relación que guarden entre sí las impedancias acústicas. La energía reflejada puede ser medida entonces en amplitud y tiempo de aparición de la señal ultrasónica.

Se utilizó un sistema de adquisición de señales ultrasónicas, que se presenta esquemáticamente en la figura 3. Se observan los componentes que conforman el sistema ultrasónico.

\section{RESULTADOS}

Primeramente se utilizó la probeta de $\mathrm{Al}-\mathrm{Pb}$, obteniéndose una señal del tiempo de recorrido del haz

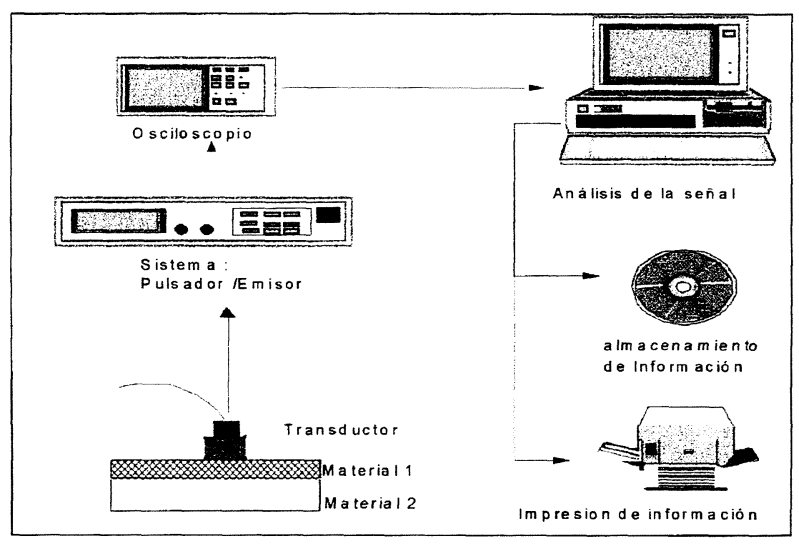

FIG. 3.- Sistema de adquisición de señales ultrasónicas

\section{FIG. 3.- System of signal acquisition ultrasonic}

ultrasónico de $3.030 \mathrm{~ms}$ correspondiente solamente al aluminio, debido a que se analizó una parte de la probeta donde no existe unión con el plomo (Fig. 4). Se procedió a analizar intencionalmente una parte defectuosa de la unión, obteniéndose la señal presentada en la figura 5. Se analizó una unión buena de la intercara $\mathrm{Al}-\mathrm{Pb}$, colocándose el transductor sobre el material de aluminio, por lo que el haz ultrasónico viajo de una baja a una alta impedancia acústica obteniéndose la señal representada en la figura 6 . Se procedió a analizar la misma unión pero ahora colocando el transductor en el material de plomo, por lo que el haz ultrasónico viajó de una alta a una baja impedancia acústica obteniéndose la señal representada en la figura 7.

Por último se analizó la unión entre Al-Fe, colocando el transductor en el aluminio, viajando el haz ultrasónico de una baja a una alta impedancia, obteniéndose la señal proporcionada en la figura 8 .

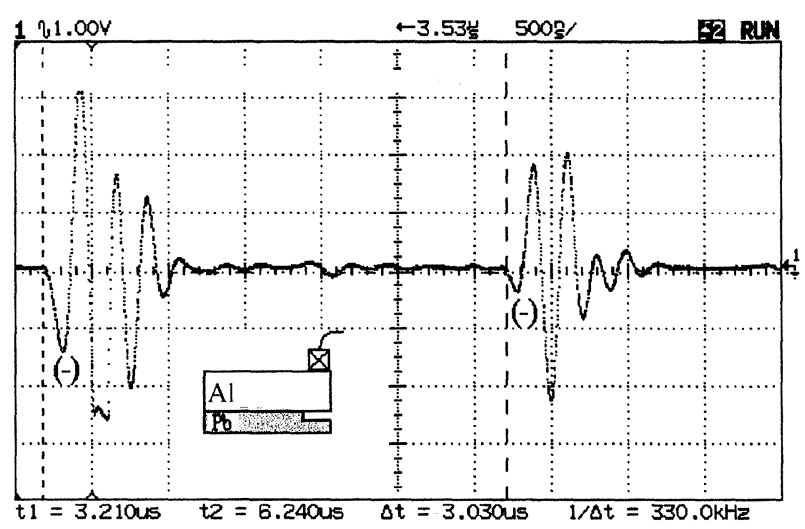

FIG 4 .- Señal ultrasónica del haz en aluminio

FIG 4.- Ultrasonic signal of the wave in aluminum 


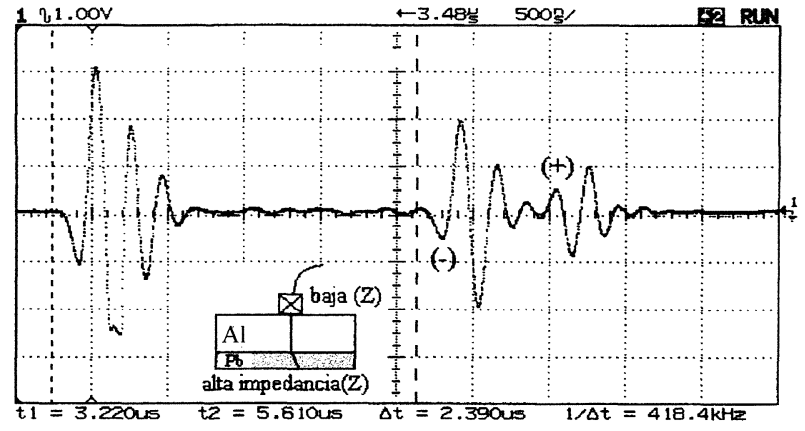

FIG 5.- Señal ultrasónica de la intercara $\mathrm{Al}-\mathrm{Pb}$ de una "unión mala".

Fig 5.-Ultrasonic signal of the Al-Pb interface of a "poor union"

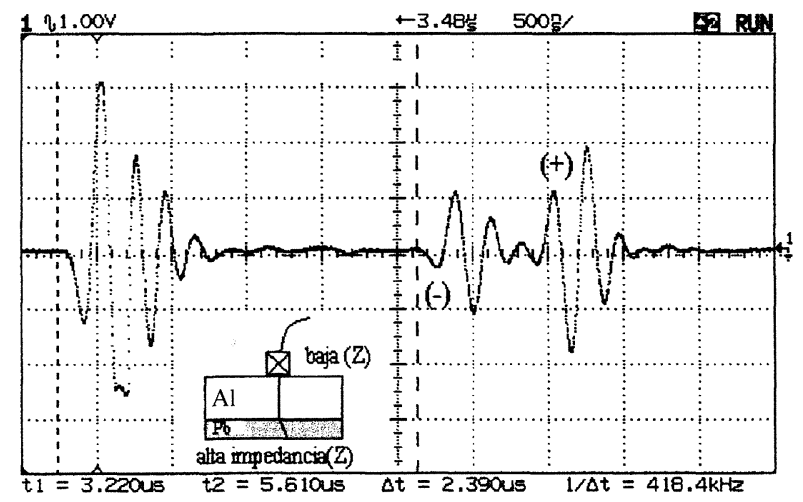

FIg 6.- Señal ultrasónica de la intercara Al-Pb de una "unión buena"

FIG 6.- Ultrasonic signal of the Al-Pb interface of a "good union"

\subsection{Análisis de resultados}

Como consecuencia del estudio realizado, se obtuvieron los siguientes resultados:

- Se observaron tres ecos en la señal ultrasónica, el primero correspondiente al eco inicial de entrada al material, el segundo a la intercara y el tercero a la pared posterior de reflexión del material.

- Se observó en la señal ultrasónica un cambio de fase, de negativa a positiva, cuando el haz ultrasónico viaja de una baja impedancia a una alta impedancia y un cambio de fase, de positiva a negativa, cuando la dirección del haz es de una alta a un baja impedancia.

- Cuando la amplitud de la señal de la intercara es mayor que la de la señal de la pared posterior, se está indicando que existe una "mala unión" y viceversa, cuando la amplitud de la señal de la intercara es menor a la de la pared posterior lo

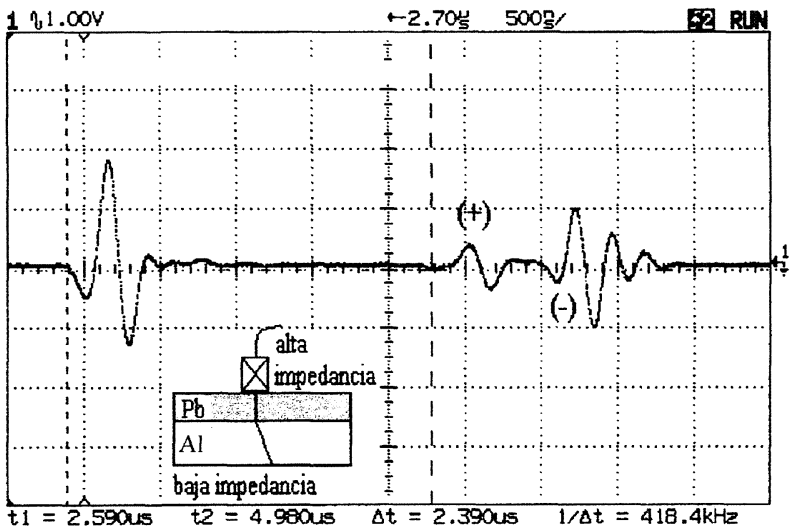

FIG 7.- Señal ultrasónica de la intercara $\mathrm{Al}-\mathrm{Pb}$ de una "unión buena".

FIG 7 .- Ultrasonic signal of the Al-Pbl interface of a "good union"

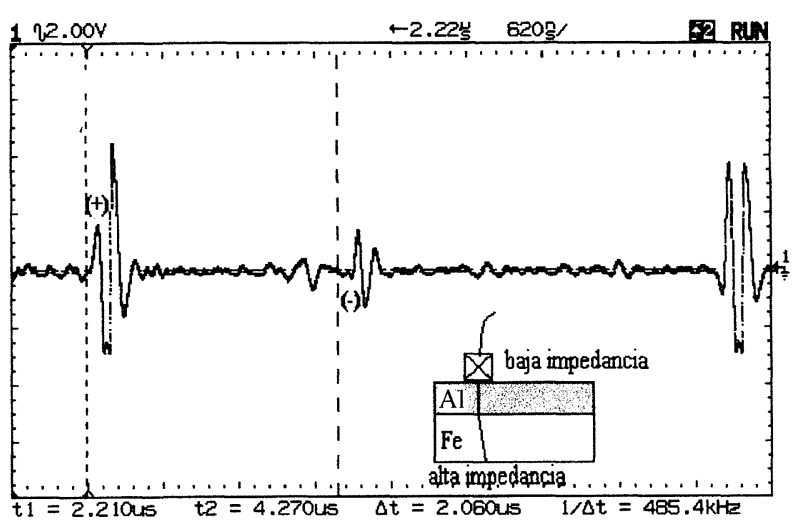

FIG 8.- Señal ultrasónica de la intercara Al-Fe de una "unión buena".

FIG 8.-Ultrasonic signal of the Al-Fe interface of a "good union"

que se indica es la existencia de una "buena unión".

- El porcentaje del coeficiente de reflexión (la relación de impedancias que guarda entre sí la unión de materiales disímiles) es la pauta para determinar el porcentaje de reflexión en que debiera ampliarse la señal de la intercara a fin de calcular cuánta menor amplitud habrá de tener respecto a la de la señal de la pared posterior para indicar que existe una "buena unión" entre esos materiales disímiles.

\section{CONCLUSIONES}

- Se concluye que la mejor manera de examinar una unión de materiales disímiles es colocando el transductor en el material de baja impedancia 
para, de esta manera, hacer que viaje el haz ultrasónico de una baja impedancia a una alta impedancia, obteniéndose así la mejor señal que demostrará si existe una "buena unión" o una "mala unión".

- La mejor forma de representación de la señal ultrasónica es la "RF" combinada con los criterios de la señal "VID" de la relación entre amplitudes de la señal de la intercara con la amplitud de la pared posterior.

- Se concluye también que se deberá tomar como pauta el porcentaje de coeficiente de reflexión para determinar en qué tanto por ciento deberá ser menor la relación entre la amplitud de la señal de la intercara y la de la pared posterior.

\section{REFERENCIAS}

(1) MClntiRe, P. Nondestructive testing handbook, 2nd ed. Vol. 7. Ed. ASNT, (U.S.A.) 1991: 341-342

(2) Jaramillo, D. Memorias del VIII Congreso Nacional de Soldadura. Morelia, Mich., México, 1994: 152-168.

(3) Davis, J.R. Metals Handbook, Ninth Edition. Vol. 17. Nondestructive Evaluation and Quality Control. Ed. ASM (U.S.A.), 1989: 234-235 